\title{
Concerning the Reactivity of Dioxiranes. Observations from Experiments and Theory
}

\author{
Cosimo Annese, ${ }^{\dagger}$ Lucia D'Accolti,${ }^{\dagger}$ Anna Dinoi, ${ }^{\dagger}$ Caterina Fusco, ${ }^{\S}$ \\ Remo Gandolfi, ${ }^{*} *$ and Ruggero Curci $i^{\dagger} *$
}

Contribution from Dipartimento Chimica, Università di Bari, CNR.-ICCOM, v. Amendola 173, I-70126 Bari, Italy and Dipartimento di Chimica Organica, Università di Pavia, v.le Taramelli 10, 27100 Pavia Italy.

E-mail: curci@ba.iccom.cnr.it

$\dagger$ University of Bari
University of Pavia
${ }^{\S}$ C.N.R.- Istituto di Chimica dei Composti Organometallici (ICCOM), Bari Section, Italy

(18 pages, including this cover)

\section{Table of Contents}

$\begin{array}{lll}\text { 1. Supplemental characterization of epoxides 5a-f } & \text { p. S2 }\end{array}$

2. Sample HPLC data for substrates and products pp. S4-8

3. Sample evaluation of relative Rates p. S9

4. Hammett plots of kinetics data p.S 10

5. Sample evaluation of absolute Rates pp. S 11-12

6. Energy of dioxiranes and dioxirane radical anions p.S 13

7. Energy, cartesian coordinates, and imaginary frequencies for all TS's p.S 14

$\begin{array}{lll}\text { 8. Supplemental References } & \text { p.S } 18\end{array}$

N.B.: Additional references, not quoted in the article, are reported in Italics. 


\section{Supplemental characterization of epoxides 5a-f}

(E)-3-(4-methoxy-phenyl)-oxirane-2-carbonitrile (5a): ${ }^{l a}$ colorless liquid, bp $155-158{ }^{\circ} \mathrm{C} / 6 \mathrm{mmHg}$ (lit. $\left.{ }^{l b} 156-157^{\circ} \mathrm{C} / 6 \mathrm{mmHg}\right) ;{ }^{1} \mathrm{HNMR}\left(\mathrm{CDCl}_{3}, 500 \mathrm{MHz}\right): \delta 7.18(\mathrm{~d}, J=9.0 \mathrm{~Hz}, 2 \mathrm{H}), 6.90(\mathrm{~d}, J=9.0$ $\mathrm{Hz}, 2 \mathrm{H}), 4.23(\mathrm{~d}, J=2.0 \mathrm{~Hz}, 1 \mathrm{H}), 3.81(\mathrm{~s}, 3 \mathrm{H}), 3.41(\mathrm{~d}, J=2.0 \mathrm{~Hz}, 1 \mathrm{H}) ;{ }^{13} \mathrm{CNMR}\left(\mathrm{CDCl}_{3}, 125 \mathrm{MHz}\right)$ : $\delta$ 160.7, 127.1, 124.4, 116.1, 114.3, 58.4, 55.3, 44.5; GC/MS (70 eV) m/z (rel. intensity): 175 (M+, 39), 146 (20), 121 (41), $104(14), 91$ (64), 77 (56), 63 (32), 51 (100).

(E)-3-(4-methyl-phenyl)-oxirane-2-carbonitrile (5b): ${ }^{l c}$ colorless oil; ${ }^{1} \mathrm{HNMR}\left(\mathrm{CDCl}_{3}, 500 \mathrm{MHz}\right)$ : $\delta 7.20(\mathrm{~d}, J=8.0 \mathrm{~Hz}, 2 \mathrm{H}), 7.15(\mathrm{~d}, J=8.0 \mathrm{~Hz}, 2 \mathrm{H}), 4.25(\operatorname{broad~s}, 1 \mathrm{H}), 3.40(\mathrm{~d}, J=2.0 \mathrm{~Hz}, 1 \mathrm{H}), 2.36$ $(\mathrm{s}, 3 \mathrm{H}) ;{ }^{13} \mathrm{CNMR}\left(\mathrm{CDCl}_{3}, 100 \mathrm{MHz}\right): \delta 139.9,129.61,129.59,125.6,116.1,58.5,44.6,21.3$; GC/MS (70 eV) m/z (rel. intensity): $159\left(\mathrm{M}^{+}, 44\right), 144$ (41), 131 (44), 116 (74), 104 (96), 91 (89), 78 (100), 65 (46), $51(96)$.

(Z)-3-(4-methyl-phenyl)-oxirane-2-carbonitrile (5b'): ${ }^{1 c}$ colorless oil; ${ }^{1} \mathrm{HNMR}\left(\mathrm{CDCl}_{3}, 500 \mathrm{MHz}\right): \delta$ $7.28(\mathrm{~d}, J=8.0 \mathrm{~Hz}, 2 \mathrm{H}), 7.22(\mathrm{~d}, J=8.0 \mathrm{~Hz}, 2 \mathrm{H}), 4.21(\mathrm{~d}, J=4.0 \mathrm{~Hz}, 1 \mathrm{H}), 3.76(\mathrm{~d}, J=4.0 \mathrm{~Hz}, 1 \mathrm{H})$, $2.36(\mathrm{~s}, 3 \mathrm{H}) ;{ }^{13} \mathrm{CNMR}\left(\mathrm{CDCl}_{3}, 125 \mathrm{MHz}\right): \delta 139.7,129.4,129.3,126.1,116.4,57.7,45.1,21.8$; GC/MS (70 eV) m/z (rel. intensity):159 ( $\left.\mathrm{M}^{+}, 28\right), 144$ (22), 131 (27), 116 (60), 104 (76), 91 (77), 78 (97), 63 (48), $51(100)$.

(E)-3-phenyl-oxirane-2-carbonitrile $(\mathbf{5 c}):{ }^{l c}$ colorless liquid, bp 135-137 ${ }^{\circ} \mathrm{C} / 16 \mathrm{mmHg}\left(\right.$ lit. $^{l d} 133-134$ $\left.{ }^{\circ} \mathrm{C} / 16 \mathrm{mmHg}\right) ;{ }^{1} \mathrm{HNMR}\left(\mathrm{CDCl}_{3}, 500 \mathrm{MHz}\right): \delta 7.40(\mathrm{~m}, 3 \mathrm{H}), 7.28$ (m, $\left.2 \mathrm{H}\right), 4.29(\mathrm{~s}, 1 \mathrm{H}), 3.42(\mathrm{~s}, 1 \mathrm{H})$; ${ }^{13} \mathrm{CNMR}\left(\mathrm{CDCl}_{3}, 125 \mathrm{MHz}\right): \delta 132.6,129.7,128.9,125.6,115.9,58.4,44.6 ; \mathrm{GC} / \mathrm{MS}(70 \mathrm{eV}) \mathrm{m} / z(\mathrm{rel}$. intensity): $145\left(\mathrm{M}^{+}, 8\right), 117(55), 90$ (100), 77 (19), 63 (21), 51 (42).

(Z)-3-phenyl-oxirane-2-carbonitrile $\left(\mathbf{5 c}^{\prime}\right):{ }^{l c}$ white solid, mp 55.5-56 ${ }^{\circ} \mathrm{C}$ (lit. $\left.{ }^{l e} 55-57{ }^{\circ} \mathrm{C}\right) ;{ }^{1} \mathrm{HNMR}$ $\left(\mathrm{CDCl}_{3}, 500 \mathrm{MHz}\right): \delta 7.42(\mathrm{~m}, 5 \mathrm{H}), 4.25(\mathrm{~d}, J=4 \mathrm{~Hz}, 1 \mathrm{H}), 3.79(\mathrm{~d}, J=4 \mathrm{~Hz}, 1 \mathrm{H}) ;{ }^{13} \mathrm{CNMR}\left(\mathrm{CDCl}_{3}\right.$, 
$125 \mathrm{MHz}): \delta$ 131.3, 129.7, 128.6, 126.2, 115.0, 57.7, 45.1; GC/MS (70 eV) m/z (rel. intensity): 145 $\left(\mathrm{M}^{+}, 11\right), 117$ (46), 90 (100), 77 (21), 63 (37), 51 (69).

(E)-3-(4-chloro-phenyl)-oxirane-2-carbonitrile (5d): ${ }^{l c}$ white solid, mp 67-68 ${ }^{\circ} \mathrm{C}\left(\right.$ lit. $^{1 c}$ 68-69 $\left.{ }^{\circ} \mathrm{C}\right)$; ${ }^{1} \mathrm{HNMR}\left(\mathrm{CDCl}_{3}, 500 \mathrm{MHz}\right):{ }^{1 c} \delta 7.37(\mathrm{~d}, J=8.0 \mathrm{~Hz}, 2 \mathrm{H}), 7.22(\mathrm{~d}, J=8.0 \mathrm{~Hz}, 2 \mathrm{H}), 4.28($ broad s, $1 \mathrm{H})$, $3.39(\mathrm{~d}, J=2.0 \mathrm{~Hz}, 1 \mathrm{H}) ;{ }^{13} \mathrm{CNMR}\left(\mathrm{CDCl}_{3}, 100 \mathrm{MHz}\right): \delta 135.8,131.2,129.2,126.9,115.7,57.8,44.6$; GC/MS (70 eV) m/z (rel. intensity):179 (M+ 44), 144 (24), 116 (63), 88 (100), 63 (35), 50 (39).

(E)-3-(3-chloro-phenyl)-oxirane-2-carbonitrile (5e): ${ }^{2}$ white solid, mp 59-61 ${ }^{\circ} \mathrm{C} ;{ }^{1} \mathrm{HNMR}\left(\mathrm{CDCl}_{3}\right.$, $500 \mathrm{MHz}): \delta 7.28(\mathrm{~m}, 2 \mathrm{H}), 7.18(\mathrm{~s}, 1 \mathrm{H}) 7.11(\mathrm{~m}, 1 \mathrm{H}), 4.20(\operatorname{broad} \mathrm{s}, 1 \mathrm{H}), 3.33(\mathrm{~d}, J=2.0 \mathrm{~Hz}, 1 \mathrm{H})$; ${ }^{13} \mathrm{CNMR}\left(\mathrm{CDCl}_{3}, 100 \mathrm{MHz}\right): \delta$ 135.1, 134.7, 130.3, 129.9, 125.5, 123.9, 115.6, 57.6, 44.5; GC/MS (70 eV) $m / z$ (rel. intensity): $179\left(\mathrm{M}^{+}, 16\right), 144$ (26), 128 (25), 116 (92), 89 (100), 75 (26), 63 (31), 50 (36).

(E)-3-(3-nitro-phenyl)-oxirane-2-carbonitrile $(\mathbf{5 f}):^{3}$ white solid, mp $118-120{ }^{\circ} \mathrm{C} ;{ }^{1} \mathrm{HNMR}\left(\mathrm{CDCl}_{3}\right.$, $500 \mathrm{MHz}): \delta 8.27(\mathrm{~d}, J=8.0 \mathrm{~Hz}, 1 \mathrm{H}), 8.16(\mathrm{~s}, 1 \mathrm{H}), 7.63(\mathrm{~m}, 2 \mathrm{H}), 4.43(\mathrm{~s}, 1 \mathrm{H}), 3.48(\mathrm{~s}, 1 \mathrm{H}) ;{ }^{13} \mathrm{CNMR}$ $\left(\mathrm{CDCl}_{3}, 125 \mathrm{MHz}\right): \delta 148.6,135.0,131.6,130.2,124.7,120.7,115.2,57.3,44.7 ; \mathrm{GC} / \mathrm{MS}(70 \mathrm{eV}) \mathrm{m} / \mathrm{z}$ (rel. intensity): $190\left(\mathrm{M}^{+}, 2\right), 173$ (11), 135 (22), 116 (12), 105 (36), 89 (100), 63 (55), 51 (45). 


\section{Sample HPLC data for substrates and products}

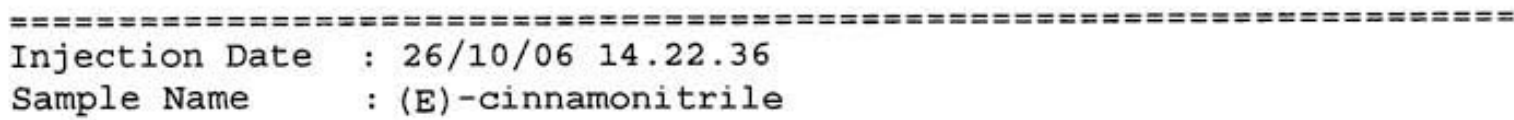

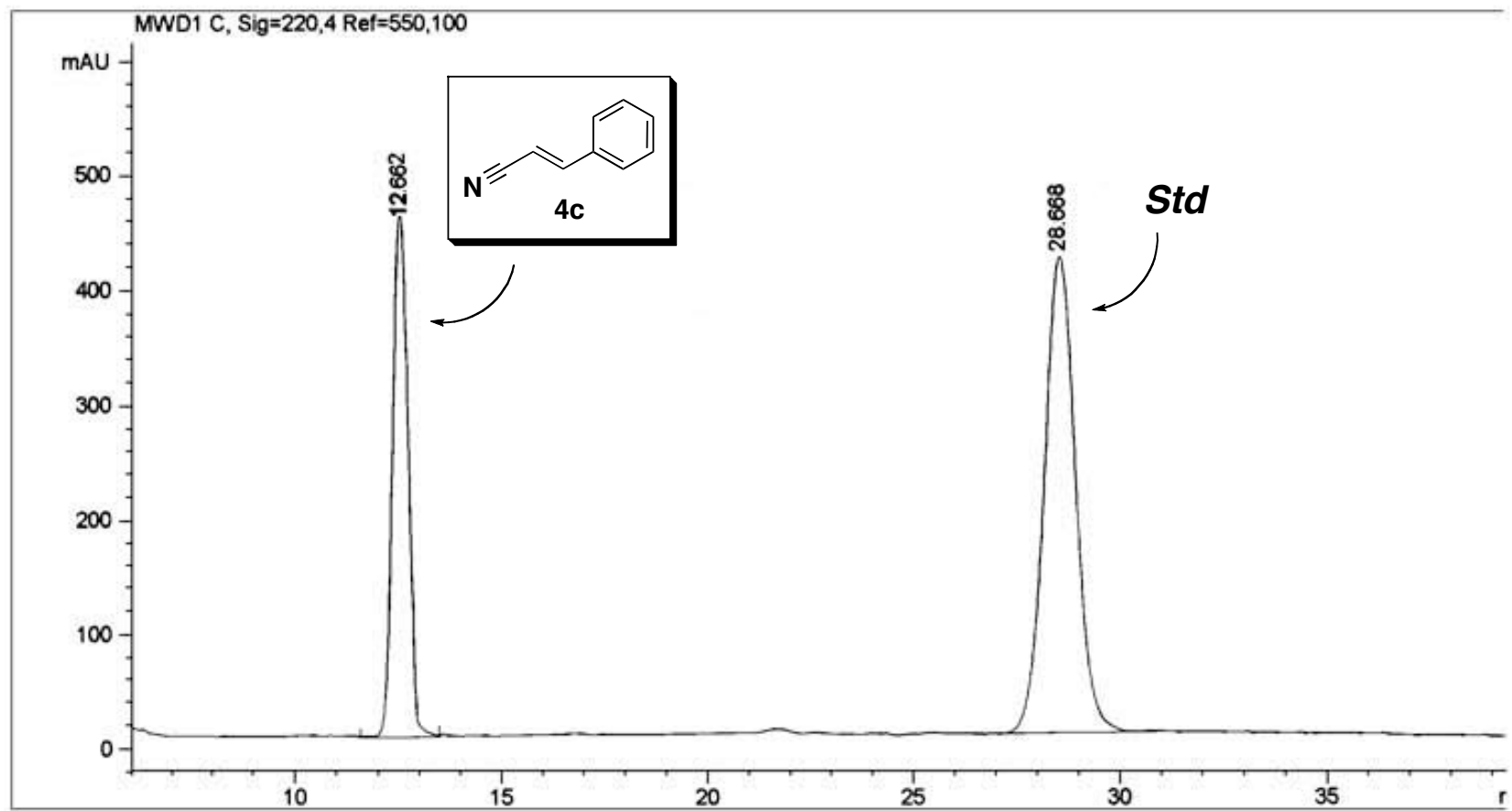

Area Percent Report

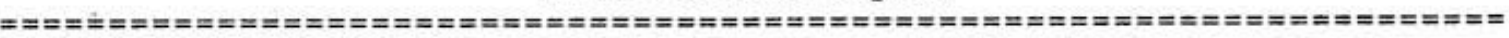

Signal 1: MWD1 C, Sig $=220,4$ Ref $=550,100$

\begin{tabular}{|c|c|c|c|c|c|c|}
\hline $\begin{array}{c}\text { Peak } \\
\text { \# }\end{array}$ & $\begin{array}{l}\text { RetTime } \\
\text { [min] }\end{array}$ & Type & $\begin{array}{l}\text { Width } \\
\text { [min] }\end{array}$ & $\begin{array}{c}\text { Area } \\
{\left[\mathrm{mAU}{ }^{*} \mathrm{~s}\right]}\end{array}$ & $\begin{array}{l}\text { Height } \\
\text { [mAU] }\end{array}$ & $\begin{array}{c}\text { Area } \\
\%\end{array}$ \\
\hline & & & & & & \\
\hline $\begin{array}{l}1 \\
2\end{array}$ & $\begin{array}{l}12.662 \\
28.668\end{array}$ & $\begin{array}{l}\text { VV } \\
\text { VB }\end{array}$ & $\begin{array}{l}0.2244 \\
0.4410\end{array}$ & $\begin{array}{l}6605.76465 \\
1.16839 \mathrm{e} 4\end{array}$ & $\begin{array}{l}457.59750 \\
414.26849\end{array}$ & $\begin{array}{l}36.1176 \\
63.8824\end{array}$ \\
\hline
\end{tabular}

Totals : $\quad 1.82896 \mathrm{e} 4 \quad 871.86600$

Results obtained with enhanced integrator!

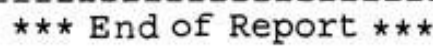

Figure 1. HPLC analysis of (E)-cinnamonitrile (4c) with methyl 2,3,4,5,6-pentafluorobenzoate $\left(\mathrm{C}_{6} \mathrm{~F}_{5} \mathrm{CO}_{2} \mathrm{Me}, \mathrm{PFMB}\right)$ internal standard. HPLC Column: Supelcosil ABZ+plus, $5 \mu \mathrm{m}(15 \mathrm{~cm} \times 4.6 \mathrm{~mm}$ id). Solvent program: $\mathrm{MeOH} / \mathrm{H}_{2} \mathrm{O} 50 / 50$, flow rate: $1.0 \mathrm{~mL} / \mathrm{min}$ : Detector: $\lambda 220 \mathrm{~nm}$. 


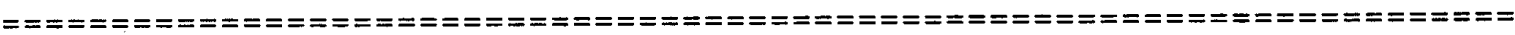

Injection Date : 27/04/06 17.23.02

Sample Name

:(E) -p-chloro-cinnamonitrile
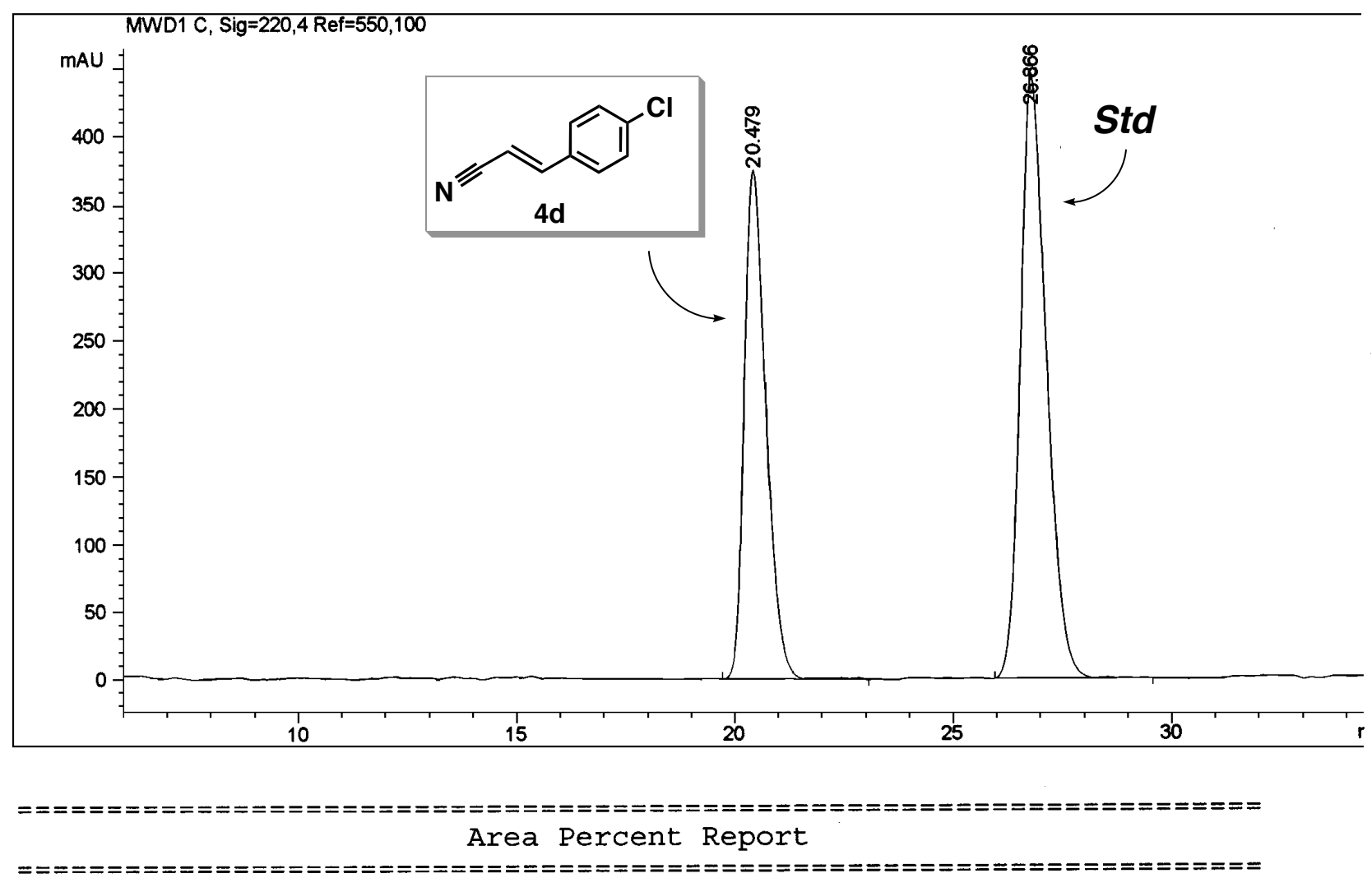

-

Signal 1: MWD1 C, Sig=220,4 Ref $=550,100$

\begin{tabular}{|c|c|c|c|c|c|c|}
\hline $\begin{array}{c}\text { Peak } \\
\quad \#\end{array}$ & $\begin{array}{l}\text { RetTime } \\
\text { [min] }\end{array}$ & Type & $\begin{array}{l}\text { Width } \\
\text { [min] }\end{array}$ & $\begin{array}{c}\text { Area } \\
{\left[\mathrm{mAU}^{*} \mathrm{~s}\right]}\end{array}$ & $\begin{array}{l}\text { Height } \\
\text { [mAU] }\end{array}$ & $\begin{array}{c}\text { Area } \\
\frac{\circ}{0}\end{array}$ \\
\hline 1 & & PP & 0 . & 1 . & 373.87592 & 9556 \\
\hline 2 & 366 & PP & 0 & $1.83525 \mathrm{e}$ & 442.48633 & 0444 \\
\hline
\end{tabular}

Totals :

$$
3.16179 e 4 \quad 816.36224
$$

Results obtained with enhanced integrator!

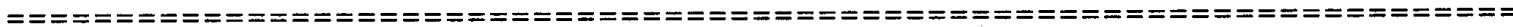

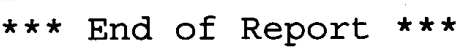

Figure 2. HPLC analysis of (E)-p-chloro-cinnamonitrile (4d). Internal standard PFMB. 


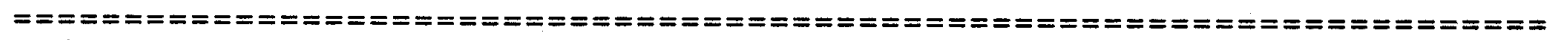

Injection Date : 26/10/06 15.49.36

Sample Name : (E)-3-phenyloxirane-2-carbonitrile

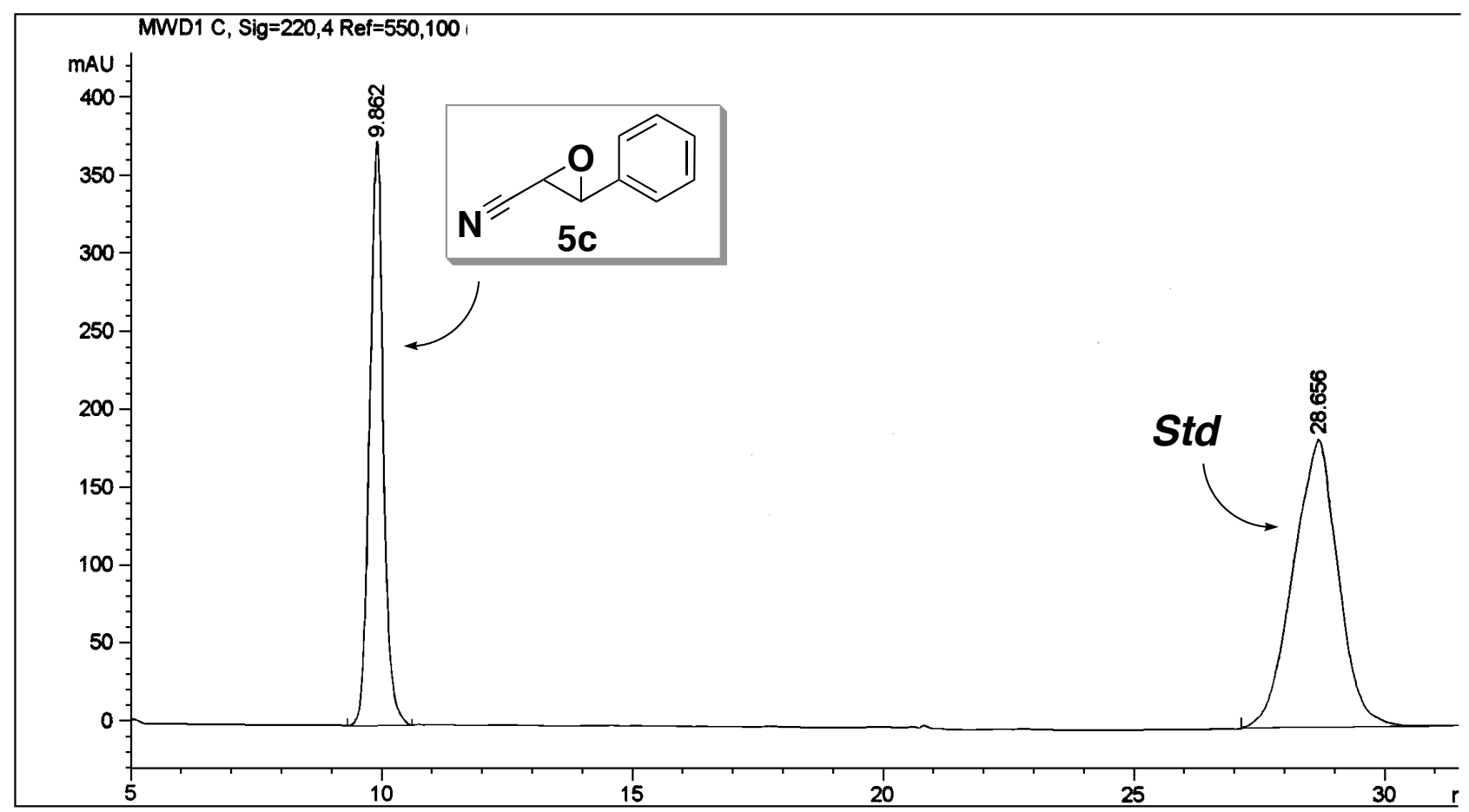

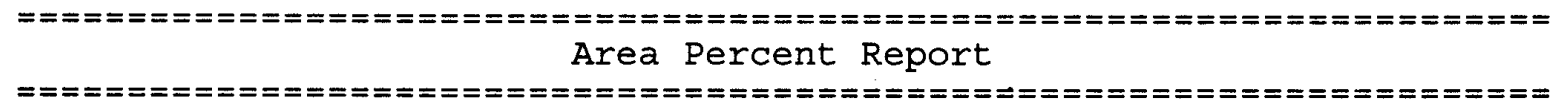

Signal 1: MWD1 C, Sig $=220,4$ Ref $=550,100$

\begin{tabular}{|c|c|c|c|c|c|c|}
\hline eak & $\begin{array}{l}\text { RetTime } \\
\text { [min] }\end{array}$ & Type & $\begin{array}{l}\text { Width } \\
\text { [min] }\end{array}$ & $\begin{array}{c}\text { Area } \\
{\left[\mathrm{mAU}^{*} \mathrm{~S}\right]}\end{array}$ & $\begin{array}{l}\text { Height } \\
\text { [mAU] }\end{array}$ & $\begin{array}{c}\text { Area } \\
\frac{\circ}{6}\end{array}$ \\
\hline$\frac{1}{2}$ & $\begin{array}{r}9.862 \\
28.656\end{array}$ & $\begin{array}{l}\text { BV } \\
\text { BBA }\end{array}$ & & $\begin{array}{l}7107.82031 \\
1.16722 \mathrm{e} 4\end{array}$ & & $\begin{array}{l}37.8477 \\
62.1523\end{array}$ \\
\hline
\end{tabular}

Totals :

$1.87800 \mathrm{e} 4 \quad 558.23305$

Results obtained with enhanced integrator!

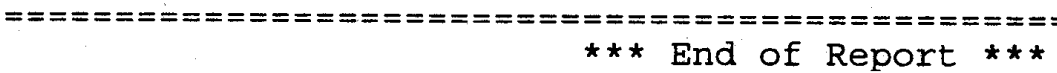

Figure 3. HPLC analysis of (E)-3-phenyl-oxirane-2-carbonitrile (5c). Internal standard PFMB. 


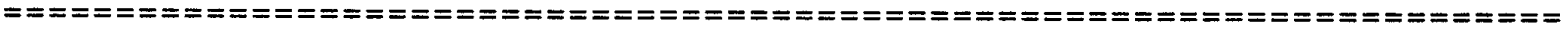

Injection Date : 27/04/06 10.23.10

Sample Name :(E)-3-(4-chloro-phenyl)-oxirane-2-carbonitrile

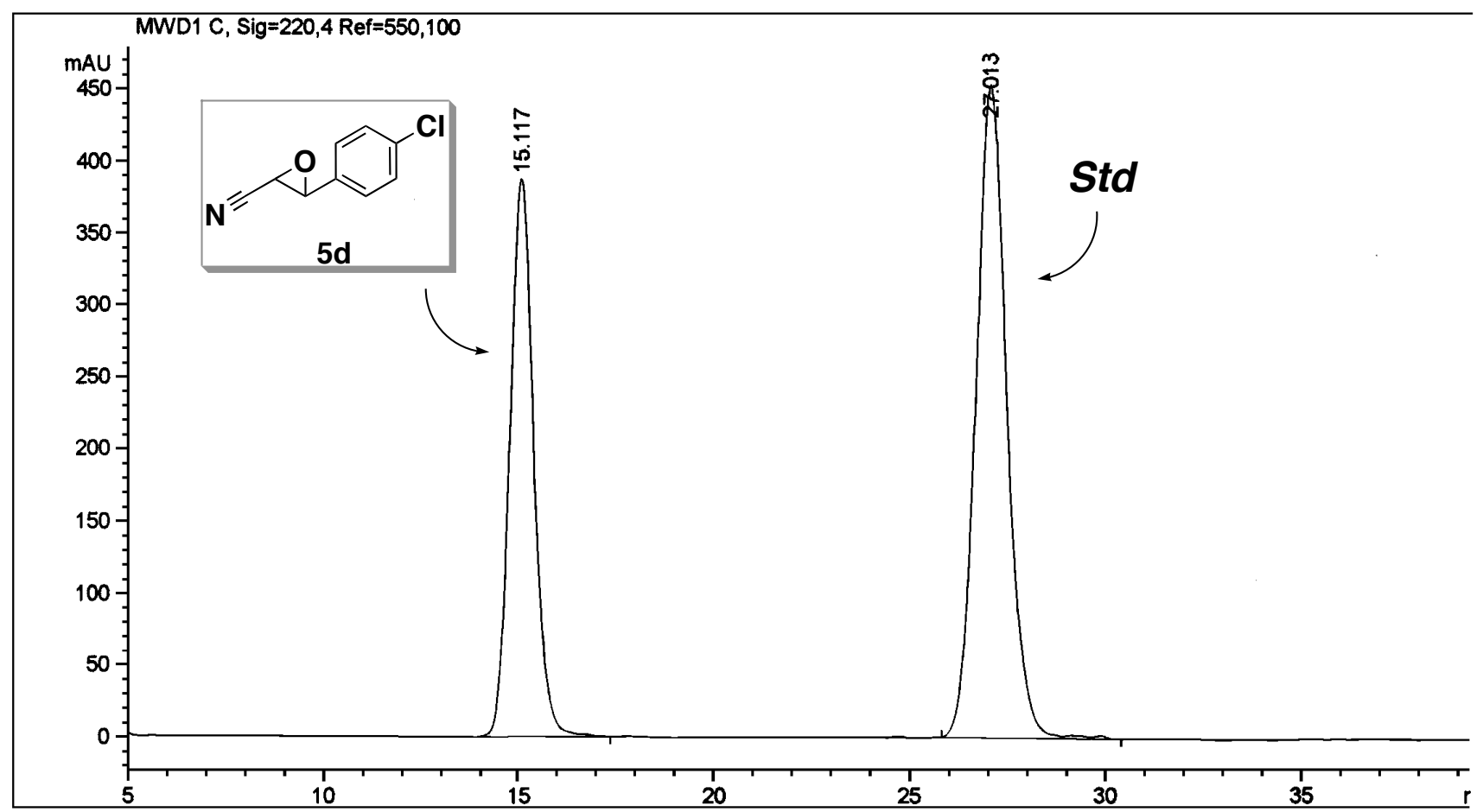

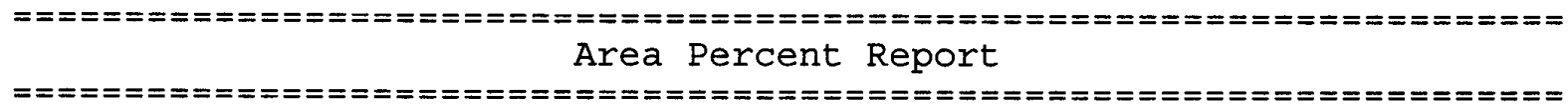

Signal 1: MWD1 C, Sig=220,4 Ref $=550,100$

\begin{tabular}{|c|c|c|c|c|c|c|}
\hline $\begin{array}{c}\text { Peak } \\
\#\end{array}$ & $\begin{array}{l}\text { RetTime } \\
\text { [min] }\end{array}$ & Type & $\begin{array}{l}\text { Width } \\
\text { [min] }\end{array}$ & $\begin{array}{c}\text { Area } \\
{\left[\mathrm{mAU}^{*} \mathrm{~s}\right]}\end{array}$ & $\begin{array}{l}\text { Height } \\
\text { [mAU] }\end{array}$ & $\begin{array}{c}\text { Area } \\
\frac{\%}{0}\end{array}$ \\
\hline $\begin{array}{l}1 \\
2\end{array}$ & $\begin{array}{l}15.117 \\
27.013\end{array}$ & $\begin{array}{l}\text { VP } \\
\text { BP }\end{array}$ & $\begin{array}{l}0.5391 \\
0.6928\end{array}$ & $\begin{array}{l}1.36754 \mathrm{e} 4 \\
2.06633 \mathrm{e}\end{array}$ & $\begin{array}{l}388.0534] \\
452.4563\end{array}$ & $\begin{array}{l}39.8251 \\
60.1749\end{array}$ \\
\hline
\end{tabular}

Totals : $\quad 3.43387 e 4 \quad 840.50980$

Results obtained with enhanced integrator!

$\star \star \star$ End of Report $* * *$

Figure 4. HPLC analysis of (E)-3-(4-chloro-phenyl)-oxirane-2-carbonitrile (5d). Internal standard PFMB. 


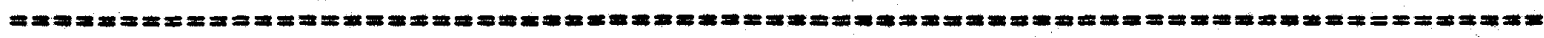

Injection Date : 27/04/06 14.18.44

Sample Name $\quad:(E)-p$-Chloro-Cinnamonitrile/ $(E)$-cinnamonitrile

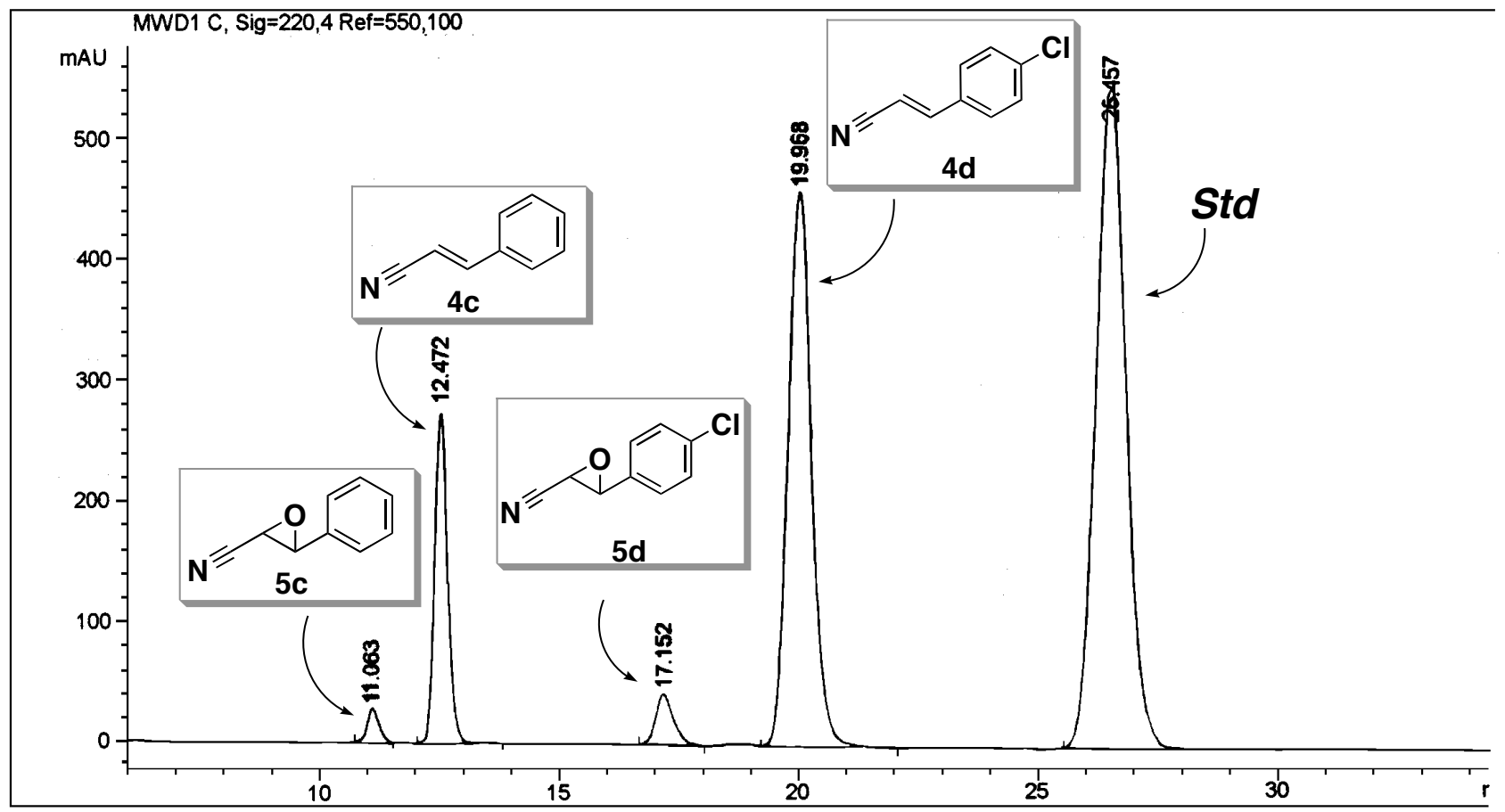

Area Percent Report

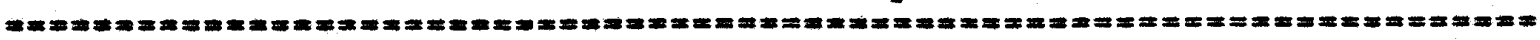

Signal 1: MWD1 C, Sig=220,4 Ref $=550,100$

\begin{tabular}{|c|c|c|c|c|c|c|}
\hline $\begin{array}{c}\text { Peak } \\
\#\end{array}$ & $\begin{array}{l}\text { RetTime } \\
\text { [min] }\end{array}$ & Type & $\begin{array}{l}\text { Width } \\
\text { [min] }\end{array}$ & $\begin{array}{c}\text { Area } \\
{[\mathrm{mAU} * \mathrm{~s}]}\end{array}$ & $\begin{array}{l}\text { Height } \\
\text { [mAU] }\end{array}$ & $\begin{array}{c}\text { Area } \\
\frac{\circ}{5}\end{array}$ \\
\hline $\begin{array}{l}1 \\
2 \\
3 \\
4 \\
5\end{array}$ & $\begin{array}{l}11.063 \\
12.472 \\
17.152 \\
19.968 \\
26.457\end{array}$ & $\begin{array}{l}\text { BV } \\
\text { PB } \\
\text { PP } \\
\text { VP } \\
\text { BP }\end{array}$ & $\begin{array}{l}0.2576 \\
0.2872 \\
0.4032 \\
0.5232 \\
0.6712\end{array}$ & $\begin{array}{l}486.37405 \\
5170.87012 \\
1144.62183 \\
1.55792 \mathrm{e} \\
2.40474 \mathrm{e} 4\end{array}$ & $\begin{array}{r}28.71361 \\
275.15204 \\
42.37085 \\
459.93002 \\
548.79407\end{array}$ & $\begin{array}{r}1.0476 \\
11.1373 \\
2.4653 \\
33.5553 \\
51.7945\end{array}$ \\
\hline \multicolumn{4}{|c|}{ Totals : } & $4.64285 e 4$ & 1354.96059 & \\
\hline
\end{tabular}

$\star \star \star$ End of Report $\star \star \star$

Figure 5. HPLC analysis of the reaction mixture resulting from competition kinetics of the TFDO (1b) oxidation of $(E)$-p-chloro-cinnamonitrile $(\mathbf{4 d})\left(\left[5.4 \times 10^{-3} \mathrm{M}\right]\right)$ and of $(E)$-cinnamonitrile $(\mathbf{4 c})\left(\left[1.15 \times 10^{-3}\right.\right.$ $\mathrm{M}])$ in acetone at $5{ }^{\circ} \mathrm{C}$. Internal standard PFMB $\left(\left[16 \times 10^{-3} \mathrm{M}\right]\right)$. HPLC column: Supelcosil ABZ+plus, 5 $\mu \mathrm{m}\left(15 \mathrm{~cm} \times 4.6 \mathrm{~mm}\right.$ id). Solvent program: $\mathrm{MeOH} / \mathrm{H}_{2} \mathrm{O} 50 / 50$, flow rate: $1.0 \mathrm{~mL} / \mathrm{min}$ : detector: $\lambda 220$ $\mathrm{nm}$. 
Table 1. Relative Rates of Epoxidation of Cinammonitrile 4b (A) vs. 4c (B) with Dioxirane 1b in Acetone at $5.0^{\circ} \mathrm{C}$.'

\begin{tabular}{ccccccc}
\hline Entry & time (s) & $\mathcal{A}^{\mathrm{A}} / \mathcal{A}^{\mathrm{St}}$ & $\begin{array}{c}10^{3}[\mathrm{~A}],{ }^{a} \\
\mathrm{M}\end{array}$ & $\mathcal{A}^{\mathrm{B}} / \mathcal{A}^{\mathrm{St}}$ & $\begin{array}{c}10^{3}[\mathrm{~B}]{ }^{a} \\
\mathrm{M}\end{array}$ & $\begin{array}{c}\text { Rel. Rate, } \\
\left(k^{\mathrm{A}} / k^{\mathrm{B}}\right)^{b}\end{array}$ \\
\hline 1 & 0 & 0.7767 & 3.29 & 2.4801 & 7.97 & - \\
2 & 23 & 0.6256 & 2.65 & 2.3743 & 7.63 & 4.96 \\
3 & 39 & 0.5265 & 2.23 & 2.2903 & 7.36 & 4.88 \\
4 & 56 & 0.4486 & 1.90 & 2.2187 & 7.13 & 4.93 \\
5 & 104 & 0.2809 & 1.19 & 2.0164 & 6.48 & 4.91 \\
\hline
\end{tabular}

${ }^{a}$ the concentration of each alkene, as estimated from the ratio of HPLC peak areas $\left(\mathcal{A}^{\mathrm{A}} / \mathcal{A}^{\mathrm{St}}, \mathcal{A}^{\mathrm{B}} / \mathcal{A}^{\mathrm{St}}\right)$, was corrected for detector response $\left(f_{\mathrm{A}}=2.597, f_{\mathrm{B}}=3.423\right)$, determined separately using standard solutions of the given alkene containing the internal reference PFMB.

${ }^{b}$ estimated as $\log \left([A] /[A]_{\mathrm{o}}\right) / \log \left([B] /[B]_{\mathrm{o}}\right)$ from the concentrations of the two competing substrates $\mathrm{A}$ and $\mathrm{B}$ at different reaction times (over the first $15-50 \%$ reaction). 
Hammett plots of kinetics data

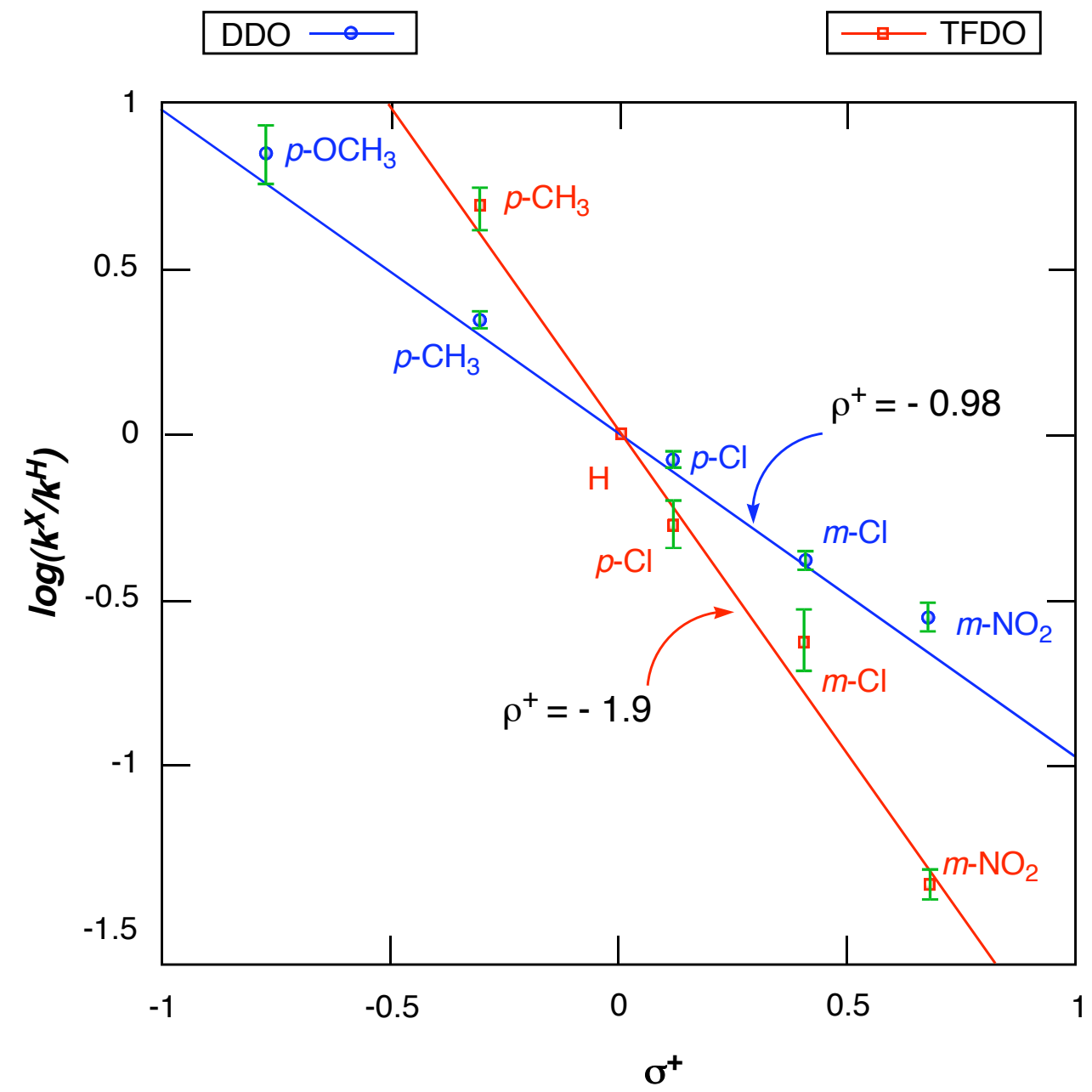

Figure 6. Hammett plots for epoxidation of substituted cinammonitriles with DDO (1a) at $25^{\circ} \mathrm{C}$ and with TFDO (1b) at $5{ }^{\circ} \mathrm{C}$, both in in acetone solvent. 


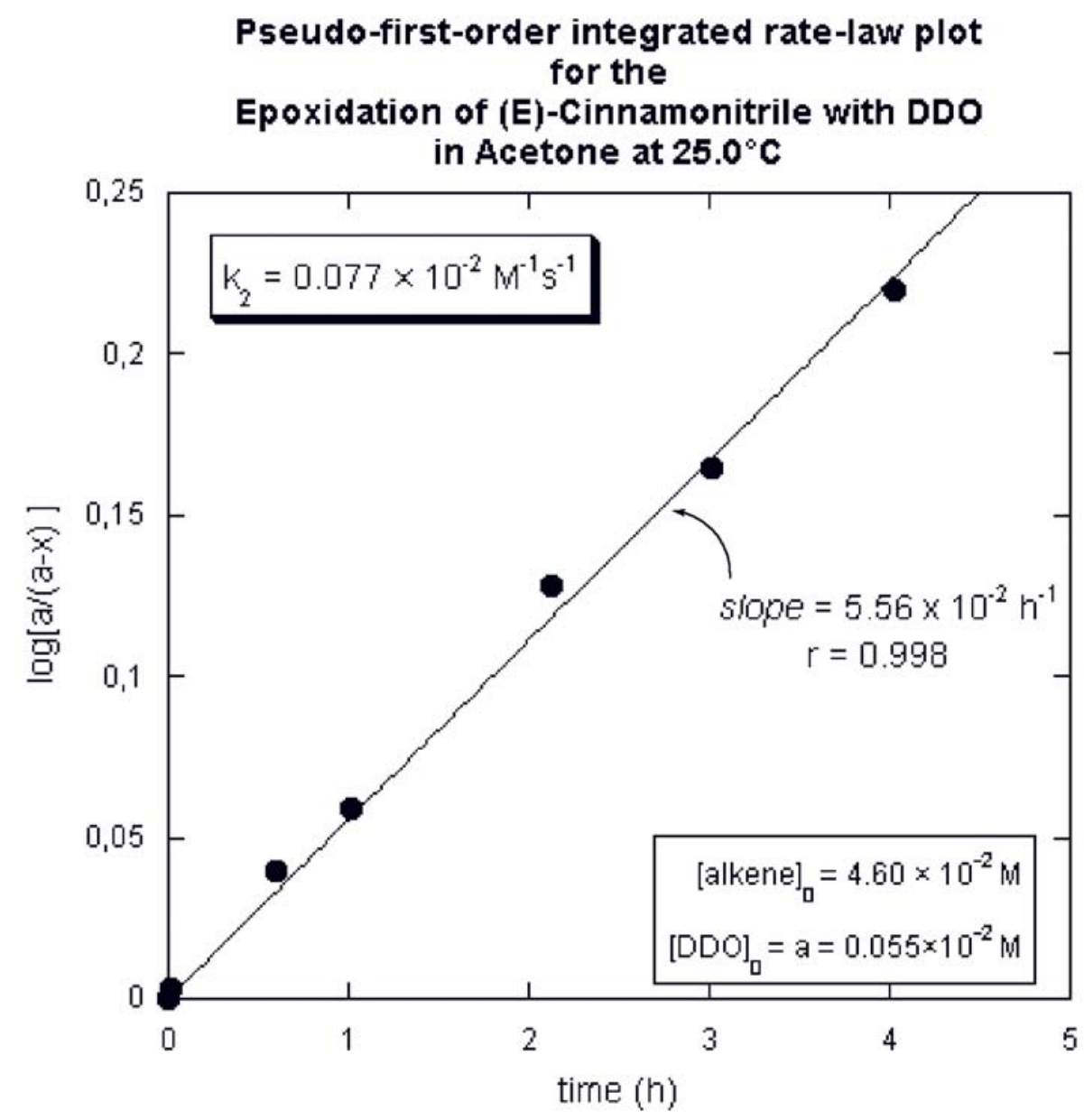

Figure 7 


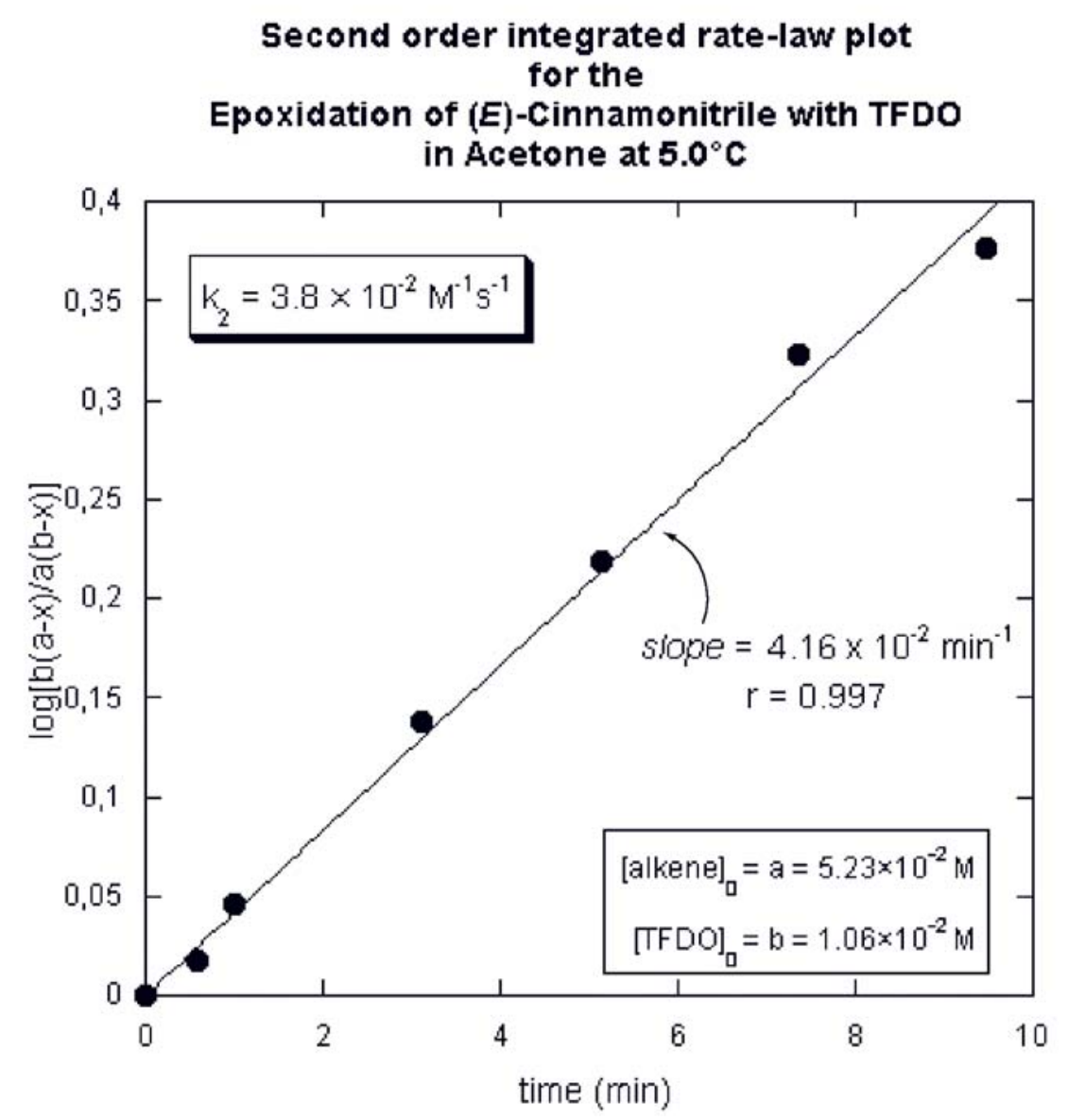

Figure 8 
Energy of dioxiranes and dioxirane radical anions at the B3LYP/6-311++G(2d,p) level (acetone and acetonitrile solution). ${ }^{4}$

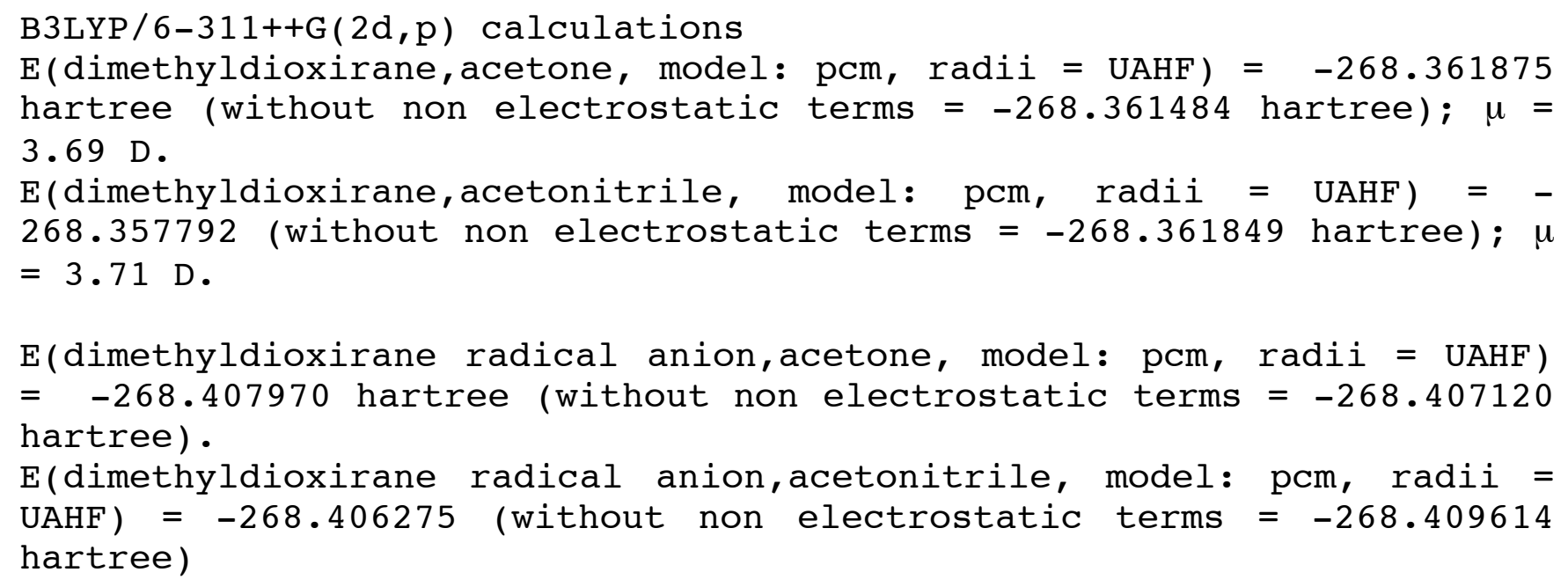

$\mathrm{E}[$ methyl (trifluoromethyl)dioxirane, acetone, model: pcm, radii = UAHF] $=-566.175873$ hartree (without non electrostatic terms $=-566.177239$ hartree); $\mu=3.13 \mathrm{D}$.

$\mathrm{E}[$ methyl(trifluoromethyl)dioxirane, acetonitrile, model: pcm, radii = $\mathrm{UAHF}]=-566.170824$ (without non electrostatic terms $=-566.177347$ hartree); $\mu=3.14 \mathrm{D}$.

E[methyl(trifluoromethyl)dioxirane radical anion, acetone, model: pcm, radii $=\mathrm{UAHF}$ ] $=-566.243683$ hartree (without non electrostatic terms $=-566.244747$ hartree) .

E[methyl(trifluoromethyl)dioxirane radical anion, acetonitrile, model: $\mathrm{pcm}, \operatorname{radii}=\mathrm{UAHF}]=-566.240785$ (without non electrostatic terms = 566.246987 hartree) 
Energy and cartesian coordinates of all TS's at the B3LYP/6-31+G(2d,p) level (gas phase and acetone solution). Imaginary frequency for all TS's. ${ }^{4}$

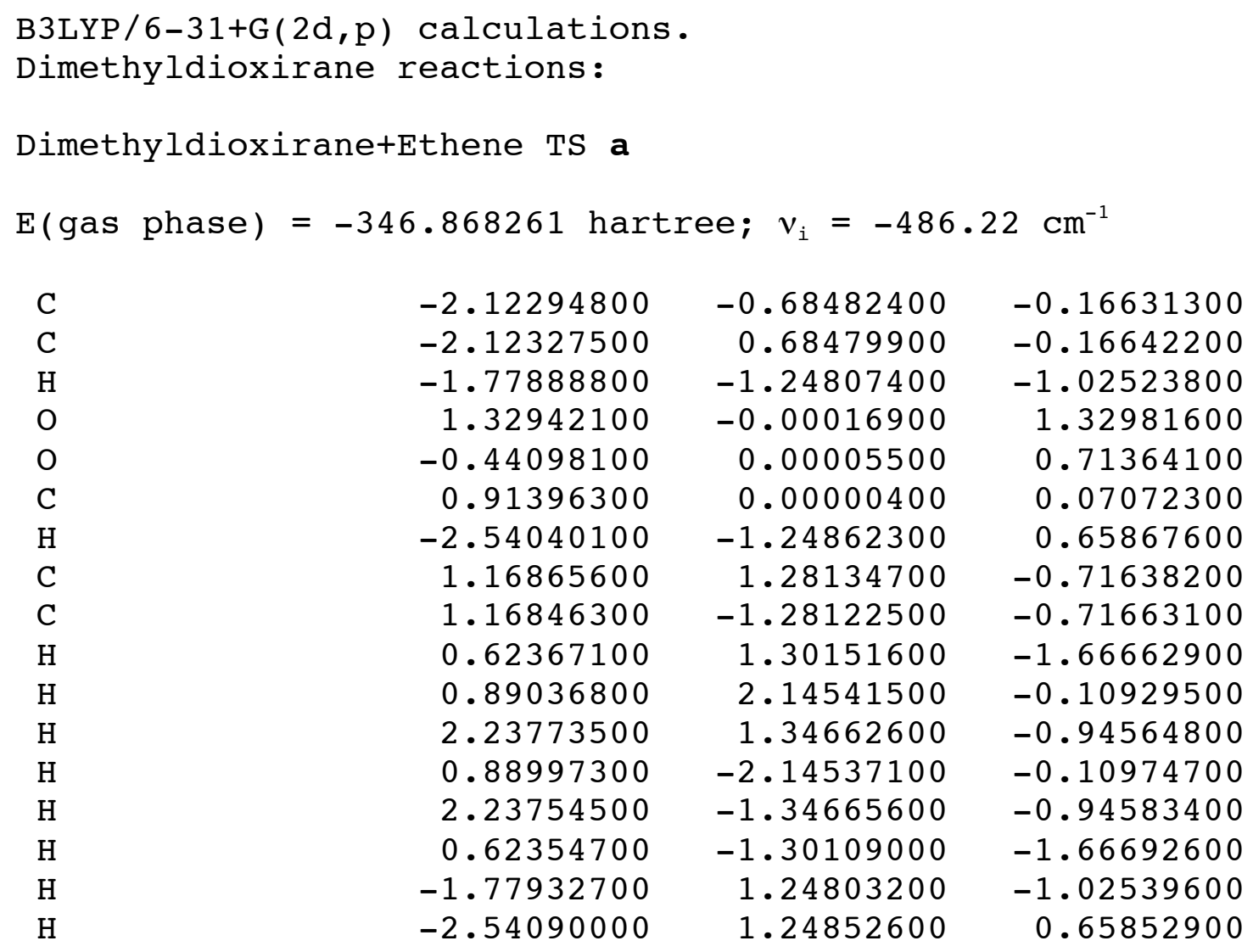

$\mathrm{E}$ (acetone; model: pcm; radii = UAHF) $=-346.875567$ hartree (without non electrostatic terms $=-346.876942$ hartree) $; v_{i}=-433.651 \mathrm{~cm}^{-1}$

$\begin{array}{lrrr}\mathrm{C} & -2.14454600 & -0.68239900 & -0.15818000 \\ \mathrm{C} & -2.14466400 & 0.68253700 & -0.15844200 \\ \mathrm{H} & -1.80593000 & -1.24672500 & -1.01928300 \\ \mathrm{O} & 1.36446800 & -0.00060400 & 1.32035500 \\ \mathrm{O} & -0.39232500 & -0.00011100 & 0.75262800 \\ \mathrm{C} & 0.90561200 & -0.00003000 & 0.05692400 \\ \mathrm{H} & -2.54968100 & -1.24645500 & 0.67362800 \\ \mathrm{C} & 1.15392900 & 1.27859100 & -0.73097600 \\ \mathrm{C} & 1.15359300 & -1.27810100 & -0.73196400 \\ \mathrm{H} & 0.58973700 & 1.29817000 & -1.66900100 \\ \mathrm{H} & 0.88809400 & 2.14665000 & -0.12346700 \\ \mathrm{H} & 2.21812600 & 1.33823500 & -0.98362100 \\ \mathrm{H} & 0.88720400 & -2.14656000 & -0.12527200 \\ \mathrm{H} & 2.21785200 & -1.33799800 & -0.98429300 \\ \mathrm{H} & 0.58977300 & -1.29663400 & -1.67023300 \\ \mathrm{H} & -1.80605200 & 1.24663600 & -1.01971100 \\ \mathrm{H} & -2.54982000 & 1.24680100 & 0.67321200\end{array}$


Dimethyldioxirane+acrylonitrile TS c

$E($ gas phase $)=-439.112127$ hartree; $v_{i}=-493.87 \mathrm{~cm}^{-1}$

$\begin{array}{lrrr}\mathrm{C} & 0.87102500 & 1.41125500 & 0.07742300 \\ \mathrm{C} & 1.60917000 & 0.49741500 & -0.65853500 \\ \mathrm{H} & 0.18064700 & 2.06897800 & -0.43405700 \\ \mathrm{O} & -1.77013200 & -0.50036000 & 1.37344700 \\ \mathrm{O} & -0.18796300 & 0.05513700 & 0.62152700 \\ \mathrm{C} & -1.54120600 & -0.23257000 & 0.08046800 \\ \mathrm{H} & 1.22655800 & 1.74591200 & 1.04390800 \\ \mathrm{C} & -1.52211500 & -1.43365200 & -0.85192500 \\ \mathrm{C} & -2.25355600 & 0.99331900 & -0.47255300 \\ \mathrm{H} & -1.11182400 & -1.18000900 & -1.83493500 \\ \mathrm{H} & -0.93319800 & -2.23509900 & -0.40237900 \\ \mathrm{H} & -2.54807200 & -1.78855500 & -0.99710700 \\ \mathrm{H} & -2.18770800 & 1.82084400 & 0.23750000 \\ \mathrm{H} & -3.31078900 & 0.74376300 & -0.60855100 \\ \mathrm{H} & -1.86153900 & 1.29911900 & -1.44873500 \\ \mathrm{C} & 2.71969500 & -0.20286900 & -0.12864300 \\ \mathrm{~N} & 3.65440700 & -0.76778100 & 0.26968700 \\ \mathrm{H} & 1.33176100 & 0.26390300 & -1.68065700\end{array}$

$\mathrm{E}($ acetone; model: $\mathrm{pcm} ;$ radii $=\mathrm{UAHF})$ ) $=-439.122306$ hartree (without non electrostatic terms $=-439.124286$ hartree); $v_{i}=-388.38 \mathrm{~cm}^{-1}$

$\begin{array}{lrrr}\mathrm{C} & 0.89619100 & 1.47500600 & 0.06265700 \\ \mathrm{C} & 1.58417200 & 0.52168300 & -0.66347200 \\ \mathrm{H} & 0.19833200 & 2.12842000 & -0.44555600 \\ \mathrm{O} & -1.72164000 & -0.60371700 & 1.36025400 \\ \mathrm{O} & -0.17213400 & 0.05448300 & 0.62272200 \\ \mathrm{C} & -1.52122900 & -0.24295500 & 0.07941000 \\ \mathrm{H} & 1.25437100 & 1.79987300 & 1.03315100 \\ \mathrm{C} & -1.48577300 & -1.37880300 & -0.92889100 \\ \mathrm{C} & -2.27570500 & 0.99293200 & -0.38037500 \\ \mathrm{H} & -1.10025700 & -1.04799200 & -1.89842400 \\ \mathrm{H} & -0.86840400 & -2.19418400 & -0.54639100 \\ \mathrm{H} & -2.50509700 & -1.74885100 & -1.08334000 \\ \mathrm{H} & -2.20725200 & 1.78123600 & 0.37292000 \\ \mathrm{H} & -3.32986700 & 0.72618500 & -0.50929000 \\ \mathrm{H} & -1.91110400 & 1.36341300 & -1.34403900 \\ \mathrm{C} & 2.67424600 & -0.21035800 & -0.12611800 \\ \mathrm{~N} & 3.58313200 & -0.80746400 & 0.28362600 \\ \mathrm{H} & 1.30613200 & 0.29299700 & -1.68748400\end{array}$


Methyl(trifluoromethyl)dioxirane reactions:

Methyl(trifluoromethyl)dioxirane+ethene TS b $\mathrm{E}($ gas phase $)=-644.615285$ hartree; $v_{i}=-383.38 \mathrm{~cm}^{-1}$

$\begin{array}{lrrr}\mathrm{C} & -2.73754400 & -0.01146700 & 0.44660600 \\ \mathrm{C} & -2.26524600 & -1.18247200 & -0.07589000 \\ \mathrm{H} & -2.46125600 & 0.30989500 & 1.44413500 \\ \mathrm{O} & 0.38806100 & 1.35613300 & -1.36618500 \\ \mathrm{O} & -1.09147700 & 0.42708900 & -0.72878400 \\ \mathrm{C} & 0.20013300 & 0.80131800 & -0.17988400 \\ \mathrm{H} & -3.46189900 & 0.59361800 & -0.08495700 \\ \mathrm{C} & 1.07546400 & -0.45678100 & 0.06132700 \\ \mathrm{C} & 0.15341100 & 1.71977600 & 1.03050100 \\ \mathrm{H} & -0.52990400 & 2.54107300 & 0.80715900 \\ \mathrm{H} & 1.15196600 & 2.13020500 & 1.20505200 \\ \mathrm{H} & -0.16539000 & 1.20083800 & 1.93830400 \\ \mathrm{~F} & 0.67592500 & -1.13677800 & 1.17361600 \\ \mathrm{~F} & 1.04727100 & -1.31625300 & -0.95921300 \\ \mathrm{~F} & 2.35622900 & -0.09274800 & 0.26556200 \\ \mathrm{H} & -1.59231400 & -1.81984000 & 0.48439200 \\ \mathrm{H} & -2.58601200 & -1.53179900 & -1.04998300\end{array}$

$\mathrm{E}($ acetone; model: $\mathrm{pcm} ;$ radii $=\mathrm{UAHF})$ ) $=-644.618840$ hartree (without non electrostatic terms $=-644.622377$ hartree); $v_{i}=-388.54 \mathrm{~cm}^{-1}$

C

C

$\mathrm{H}$

O

O

$\mathrm{C}$

$\mathrm{H}$

$\mathrm{C}$

C

$\mathrm{H}$

$\mathrm{H}$

$\mathrm{H}$

F

F

F

$\mathrm{H}$

$\mathrm{H}$
$-2.80234200$

$-2.32071800$

$-2.56532000$

0.41843100

$-1.04337500$

0.20144200

$-3.49159800$

1.08433600

0.13501700

$-0.53798000$

1.13161400

$-0.21573100$

0.69958500

1.06384200

2.36642200

$-1.67717500$

$-2.59931300$
$-0.00690900$

$-1.18831700$

0.33901500

1.37600200

0.46284300

0.79450700

0.59594300

$-0.46102400$

1.68590900

2.51750400

2.08235200

1.14598400

$-1.16604500$

$-1.29729500$

$-0.08787400$

$-1.81798900$

$-1.55765400$
0.40902600

$-0.06374500$

1.40926400

$-1.33842400$

$-0.76649700$

$-0.15075500$

$-0.17102900$

0.06502300

1.07337200

0.85641100

1.28845100

1.95653300

1.15724200

$-0.97881100$

0.26674600

0.53986500

$-1.04425400$ 
Methyl(Trifluoromethyl)dioxirane+acrylonitrile TS d

$E($ gas phase $)=-736.856012$ hartree; $v_{i}=-438.59 \mathrm{~cm}^{-1}$

$\begin{array}{lrrr}\mathrm{C} & 1.36015800 & -1.36634600 & -0.35445300 \\ \mathrm{C} & 2.12761500 & -0.65824900 & 0.55007200 \\ \mathrm{H} & 0.53421000 & -1.96845100 & -0.00203700 \\ \mathrm{O} & -0.83305200 & 1.32170600 & -1.41390300 \\ \mathrm{O} & 0.54991500 & 0.27544800 & -0.74043900 \\ \mathrm{C} & -0.69862300 & 0.80301500 & -0.20135800 \\ \mathrm{H} & 1.73253100 & -1.57275200 & -1.34972100 \\ \mathrm{C} & -0.52780900 & 1.76321400 & 0.96209600 \\ \mathrm{C} & -1.70217900 & -0.33930100 & 0.10201500 \\ \mathrm{C} & 3.35956100 & -0.04456900 & 0.21048300 \\ \mathrm{~N} & 4.38614600 & 0.44313800 & -0.02981800 \\ \mathrm{H} & 1.79624700 & -0.54549900 & 1.57710000 \\ \mathrm{H} & 0.24943000 & 2.48147100 & 0.69663900 \\ \mathrm{H} & -1.46690600 & 2.30011300 & 1.12302100 \\ \mathrm{H} & -0.26269200 & 1.24978400 & 1.88980200 \\ \mathrm{~F} & -2.92343100 & 0.16907300 & 0.33190500 \\ \mathrm{~F} & -1.33705800 & -1.03113800 & 1.21859500 \\ \mathrm{~F} & -1.79874100 & -1.22354000 & -0.89544100\end{array}$

$\mathrm{E}($ acetone; model: $\mathrm{pcm} ; \operatorname{radii}=\mathrm{UAHF})$ ) $=-736.860750$ hartree (without nonelectrostatic terms $=-736.865219$ hartree); $v_{i}=-429.50 \mathrm{~cm}^{-1}$

$\mathrm{C}$
$\mathrm{C}$
$\mathrm{H}$
$\mathrm{O}$
$\mathrm{O}$
$\mathrm{C}$
$\mathrm{H}$
$\mathrm{C}$
$\mathrm{C}$
$\mathrm{C}$
$\mathrm{N}$
$\mathrm{H}$
$\mathrm{H}$
$\mathrm{H}$
$\mathrm{H}$
$\mathrm{F}$
$\mathrm{F}$
$\mathrm{F}$
1.38678400

2.17187400

0.56709600

$-0.74167800$

0.57397000

$-0.67249700$

1.70295900

$-0.53379200$

$-1.72879900$

3.37437900

4.37025700

1.90466100

0.25797500

$-1.47338100$

$-0.30244100$

$-2.95083900$

$-1.48486100$

$-1.77482200$
$-1.43649800$

$-0.62520500$

$-1.98432300$

1. 37017700

0.25612600

0.79355800

$-1.72729200$

1.68781400

$-0.32633800$

$-0.03177900$

0.44776000

$-0.42207500$

2. 41309300

2. 22402600

1.12043200

0.22012900

$-1.06370700$

$-1.17174500$
$-0.16485800$

0.62336700

0.28062400

$-1.34342800$

$-0.66319400$

$-0.14536900$

$-1.15879000$

1.07027100

0.04278100

0.15352600

$-0.20137900$

1.65676500

0.87434500

1.23282700

1.97542100

0.18566500

1.15528200

$-0.99393300$ 


\section{Supplemental References}

(1) (a) Deschamps, B.; Seyden-Penne, J. Tetrahedron 1971, 27, 3959. (b) Martynov, V. F.; Schschelkunov, A. V. J. Gen. Chem. USSR (Engl. Transl.) 1962, 32, 2348. (c) see article ref. 30a. (d) see article ref. $30 \mathrm{~b}(e)$ see ref. $30 \mathrm{c}$ in the article.

(2) see ref. 31 in the article.

(3) see ref. 32 in the article.

(4) Gaussian 03, Revision C.02, M. J. Frisch, G. W. Trucks, H. B. Schlegel, G. E. Scuseria, M. A. Robb, J. R. Cheeseman, J. A. Montgomery, Jr., T. Vreven, K. N. Kudin, J. C. Burant, J. M. Millam, S. S. Iyengar, J. Tomasi, V. Barone, B. Mennucci, M. Cossi, G. Scalmani, N. Rega, G. A. Petersson, H. Nakatsuji, M. Hada, M. Ehara, K. Toyota, R. Fukuda, J. Hasegawa, M. Ishida, T. Nakajima, Y. Honda, O. Kitao, H. Nakai, M. Klene, X. Li, J. E. Knox, H. P. Hratchian, J. B. Cross, C. Adamo, J. Jaramillo, R. Gomperts, R. E. Stratmann, O. Yazyev, A. J. Austin, R. Cammi, C. Pomelli, J. W. Ochterski, P. Y. Ayala, K. Morokuma, G. A. Voth, P. Salvador, J. J. Dannenberg, V. G. Zakrzewski, S. Dapprich, A. D. Daniels, M. C. Strain, O. Farkas, D. K. Malick, A. D. Rabuck, K. Raghavachari, J. B. Foresman, J. V. Ortiz, Q. Cui, A. G. Baboul, S. Clifford, J. Cioslowski, B. B. Stefanov, G. Liu, A. Liashenko, P. Piskorz, I. Komaromi, R. L. Martin, D. J. Fox, T. Keith, M. A. Al-Laham, C. Y. Peng, A. Nanayakkara, M. Challacombe, P. M. W. Gill, B. Johnson, W. Chen, M. W. Wong, C. Gonzalez, and J. A. Pople,Gaussian, Inc., Pittsburgh PA, 2003. See also ref. 33 in the article. 